https://www.journal-imab-bg.org

Review article

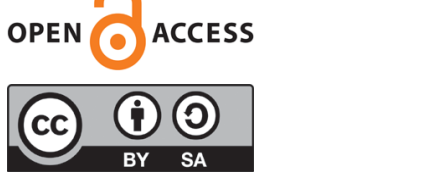

\title{
MONITORING OF VITAL SIGNS AND HEMODYNAMIC CHANGES IN PATIENTS UNDERGOING TOOTH EXTRACTION AND THIRD MOLAR SURGERY - LITERATURE REVIEW
}

\author{
Natali Tomeva, Elitsa Deliverska \\ Department of Dental, Oral and Maxillofacial Surgery, Faculty of Dental \\ Medicine, Medical University-Sofia.
}

\begin{abstract}
Background: Monitoring is global methods of observation and data recording in relation to organs and system function that afford constant information to ensure continuous evaluation of the patient's physical condition. Blood pressure heart rate, oxygen saturation and temperature are used often as an indirect measurement and determination of these parameters combined can serve as good indicators of anxiety, and pain before and during the procedure.

Purpose: The purpose of this study is to determine and analyze the changes in vital signs and hemodynamic changes induced by a dental extraction and third molar surgery.

Material and methods: Electronic searches were conducted through the MEDLINE (PubMed), Scopus etc. databases to screen all relevant articles published from inception to April 2019.

Results: All surveys included in this review were studied for vital signs and hemodynamic changes after the administration of local anesthetic at various points of time. Measurement of hemodynamic parameters showed no hypertensive peaks in systolic and diastolic blood pressure at any evaluation time. The type of local anesthetic slightly affected diastolic and systolic BP during different measurement periods without statistical significance. Pulse rate varied slightly during different measurement periods, and this variation did not achieve statistical significance. Measurement of oxygen saturation showed statistically significant differences at time of local anesthetic administration and 15 minutes after tooth extraction.

Conclusion: Monitoring during oral surgery allows the surgeon to identify immediately situations of emergency before and during the surgical procedure, establish an early diagnosis and prevent possible complications, and operate with optimized safety.
\end{abstract}

Keywords: Blood pressure, heart rate, monitoring physiologic, local anesthetic, oxygen saturation, tooth extraction,

\section{INTRODUCTION}

Basic monitoring provides essential information for assessing the principal vital signs, both circulatory and respiratory, and fundamentally comprises the control of blood pressure (BP) (through sphygmomanometry) and heart rate (HR) and rhythm. Pulsioxymetry is used to record HR and oxygen saturation $(\mathrm{SaO})$. Patient monitoring during the surgical extraction of molars is advisable when the surgical procedure is expected to be traumatic or if the patient's psychological condition makes such control desirable to optimize safety. Monitoring during oral surgery allows the surgeon to immediately identify situations of increased risk before the surgical procedure, establish an early diagnosis and prevent possible complications, and operate with increased safety. The advantages of monitoring are the ability to detect, evaluate, and prevent emergency situations in clinical practice. [1]

Tooth extraction is one of the most feared dental procedures by patients. Local anesthesia causes loss of painful sensation from a localized area of the body without inducing a loss of consciousness, by reversibly blocking nerve conduction. Use of safe and efficient local anesthetic agent and local anesthesia technique has evolved with time making the procedure more patient- friendly. Hemodynamic changes during tooth extraction can be attributed to both stresses during the procedure and presence of the vasoconstrictor, epinephrine in the local anesthetic solution. Epinephrine is added to the local anesthesia to slow the systemic absorption of local anesthetic, thus prolonging the action and intensity of the block. Also, the use of vasoconstrictor increases safety by lowering the required anesthetic dose and improve local control of bleeding. Monitoring hemodynamic changes during extraction allow the dentist to identify immediately situations of increased risk, establish an early diagnosis, prevent possible complications and operate with increased safety. Oral surgical procedures are very likely to induce patient stress, resulting in the release of endogenous catecholamines and giving rise to small hemodynamic fluctuations rather than epinephrine in the local anaesthetic solution. Stressful dental procedures can provoke a reaction in the sympathetic nervous system that could lead to hemodynamic changes. 


\section{Local anesthetic and local anaesthesia}

Several studies last few years evaluated the hemodynamic changes associated with $4 \%$ articaine with 1 : 100,000 epinephrine. [2,3]

They describe that administration of $4 \%$ articaine with 1:100,000 epinephrine was safe during tooth extraction procedure with no statistical difference in haemodynamic status between healthy patients and patients with controlled hypertention.

It has been discussed as well that tooth avulsion is the most critical point of the extraction procedure in terms of hemodynamic alterations $[2,3]$.

\section{Transient Loss of Consciousness}

Transient Loss of Consciousness (TLOC) also known as Vasovagal Reflex (VVR), which in this case is most probably of non-cardiac subtype, has been reported to occur upon extraction. More recently, Trigeminocardiac Reflex (TCR) has also been suggested as the possible mechanism of critical hemodynamic alterations upon extraction [3]. Different authors have found that in healthy normotensive patients, slight elevations of Systolic Blood Pressure (SBP) and Diastolic Blood Pressure (DBP) occur up to the moment of extraction followed by a decreased SBP and DBP to levels even lower than those recorded at baseline $[2,3]$. This is thought to be attributable to the release of endogenous adrenaline as a reaction to the noxus. Generally, Heart Rate (HR) and Blood Pressure (BP) and other clinical hemodynamic alterations throughout the extraction procedures are also a factor of patient age, gender, pain perception, psychological factors, and systemic conditions as well as local anesthetics, and the procedure itself among other factors.

While clinicians have been advised to take into account monitoring and managing the hemodynamic alterations during dental procedures, the body of literature on the topic remains to be inconclusive and scarce [2, 3]. For example, while VVR has been suggested as the cause of dental office syncope during extraction, studies have not always shown the biphasic nature of the reflex. There-fore, in the present study, the authors have attempted to revisit the extraction bradycardia in a prospective cohort of normotensive, otherwise-healthy patients and discuss the findings within the contemporary literature in the con-text of the current (pathophysiological) knowledge. [3]

\section{General considerations}

In order to avoid potentially serious reactions, dentists are obligated to monitor continuously their medically challenged patients. Monitoring of medically challenged patients should include: the metabolism of important tissues to ensure their individual health, and the function of these tissues to ensure that their contributions to the body as a whole are being maintained. Practically, there is no method for doing this in the dental setting. What can be assessed are gross neurologic function and several basic processes involved with the delivery of oxygen and essential nutrients to the tissues, including the removal of car- bon dioxide and other waste products. These functions often result in signals (sounds, pressure changes, electrical currents, spectrophotometric differences) that can be accurately measured. Monitoring methods differ greatly in cost, invasiveness, reliability, and size. Some monitoring techniques are suitable for routine use in the medically challenged dental patient, and others are best reserved for special situations. [4]

\section{Verbal contact}

In the conventional office- based dentistry, maintaining verbal contact is essential to monitoring central nervous system function of the patient. Conversation requiring simple, quick responses may be conducted during the appointment without interrupting dental treatment. Unfortunately, dentists concentrating on the procedures they are performing may neglect to communicate with their patients. Coma scales (e.g., the Japan Coma Scale or the Glasgow Coma Scale) are often used in medicine for evaluation of consciousness; in dentistry, simple verbal contact is normally sufficient to monitor the adequacy of central nervous system function in the outpatient setting. [4]

\section{Pulse oximetry (SpO2)}

Pulse oximetry is a valuable measure of arterial blood oxygenation and has become a standard monitor whenever parenteral sedation or general anaesthesia is administered. The pulse oximeter uses two light diodes, one producing visible red light at $660 \mathrm{~nm}$, and the other infrared radiation at $940 \mathrm{~nm}$. Visible red light is absorbed more by reduced haemoglobin than by oxyhaemoglobin, whereas the reverse relationship holds with the infrared wavelength. Because oxygen bound to haemoglobin normally constitutes about $98 \%$ of all oxygen in the blood, pulse oximetry provides an accurate indication of the total amount of oxygen available for delivery to tissues and generally yields a good estimate of the arterial oxygen tension $(\mathrm{PaO} 2)$.

Fig. 1. Pulse oximeter

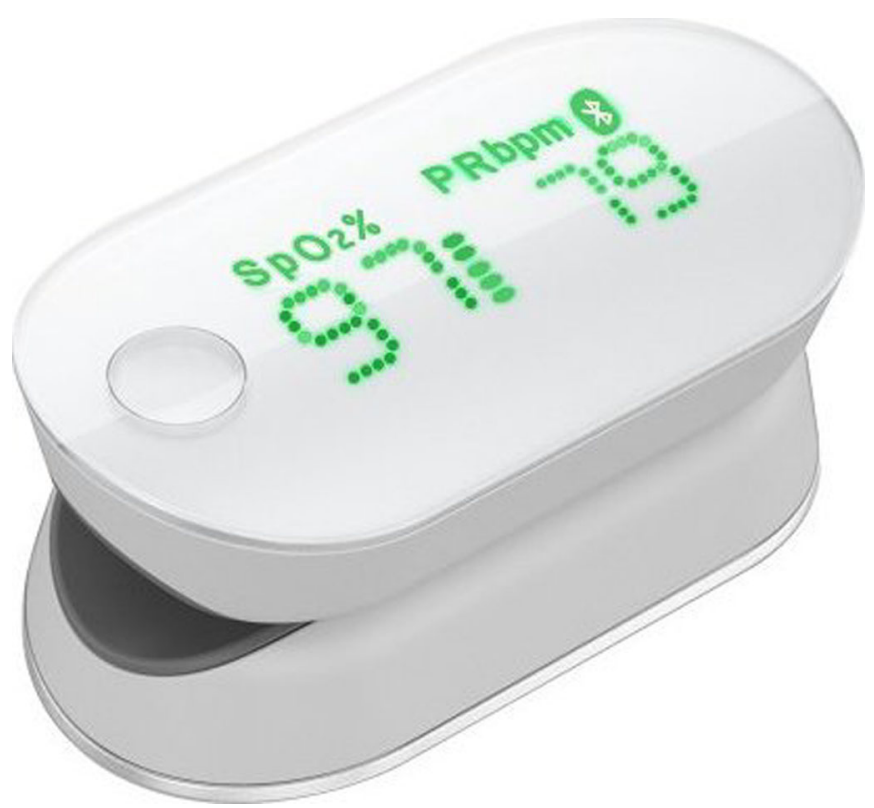


Although highly reliable, pulse oximetry is subject to certain variables that can influence measurements. Movements causing venous pulsations can confuse the pulse oximeter into recording extra "beats" of poorly oxygenated blood. Inadequate peripheral pulses because of low blood pressure, peripheral vasoconstriction (from peripheral vascular disease, high anxiety, or cold ambient temperatures), or physical obstruction (pressure occlusion of the arterial supply) may preclude effective measurement. [5]

The perfusion index (PI) is derived from the photoelectric plethysmographic signal of a pulse oximeter and is calculated as the ratio of the pulsatile component (arterial compartment) and the nonpulsatile component (other tissues; venous blood, bone, connective tissue) of the light reaching the device's detector. PI has been used as a marker of peripheral perfusion. Lima et al. [5] reported that low PI was associated with low peripheral perfusion. De Felice et al. [6] reported that the PI was a useful predictor of high illness severity in neonates. (Fig. 2)

Fig. 2. Pulse oximetry with plethysmograph and perfusion index
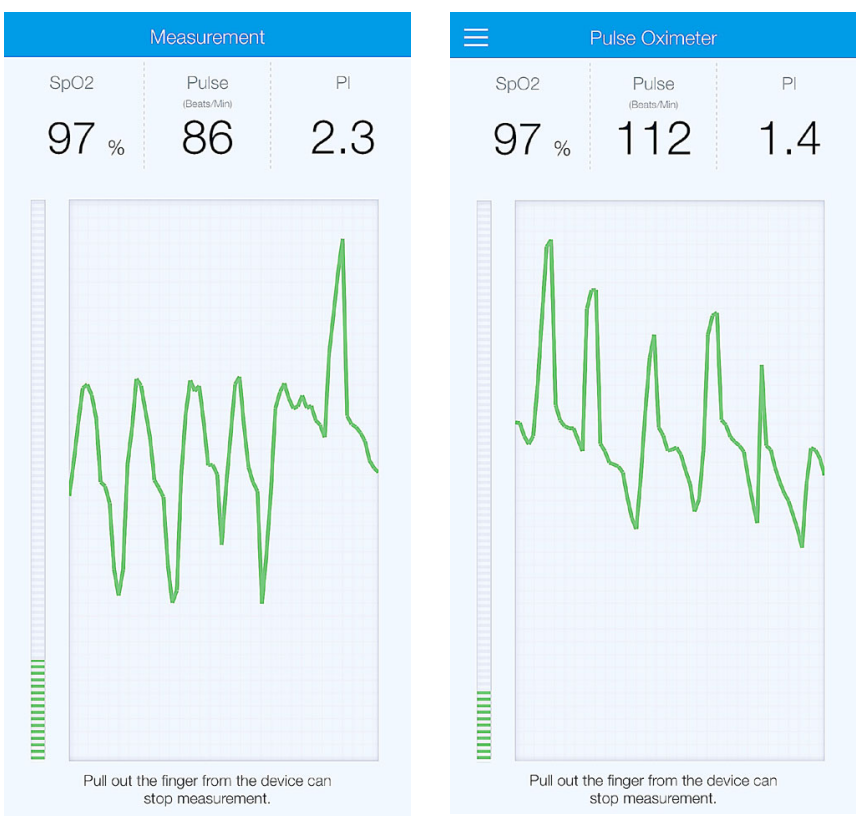

\section{Blood pressure}

Blood pressure is a vital sign that must be evaluated in medically challenged patients. Two basic factors govern the blood pressure: the cardiac output and the peripheral vascular resistance. In turn, the cardiac output is a product of the heart rate and stroke volume, and the peripheral resistance is a function of the diameter and compliance of the vasculature and the viscosity of the blood. Alterations in both cardiac output and peripheral resistance may occur in response to the stress of dental treatment. Certain medical emergencies such as vasovagal syncope (fainting) and hypertensive crises are closely related to changes in blood pressure. Table 1 classifies blood pressure for adults according to standards published by the United States National Heart, Lung, and Blood Institute along with general recommendations for dental care. [7, 8].
The most basic monitor to measure blood pressure is the manual sphygmomanometer. Each dental office should have a sphygmomanometer in good working order, and every dentist should know how to measure the blood pressure and use the data in developing an optimal treatment plan for the patient.

Automated blood pressure monitors are commonly used in dental settings. Most of these devices can measure the blood pressure automatically (i.e., without having to inflate the cuff manually) at preset intervals (i.e., every few minutes). They provide reasonably accurate data compared to manual sphygmomanometers and are preferred in patients with hypotension. Since a small computer is usually incorporated in such machines, they can often save, output, and print the data on command.

Continuous blood pressure monitoring can be obtained using arterial catheterisation, typically of the radial artery in the wrist or the dorsal artery of the foot. Artery catheterisation also permits the sampling of arterial blood for blood gas analysis $(\mathrm{PaO} 2, \mathrm{PaCO} 2, \mathrm{pH}$, bicarbonate, etc.). However, as it is painful, invasive, and must be performed under sterile conditions, it is not used in officebased dental practice.

Fig. 3. Digital sphygmomanometer

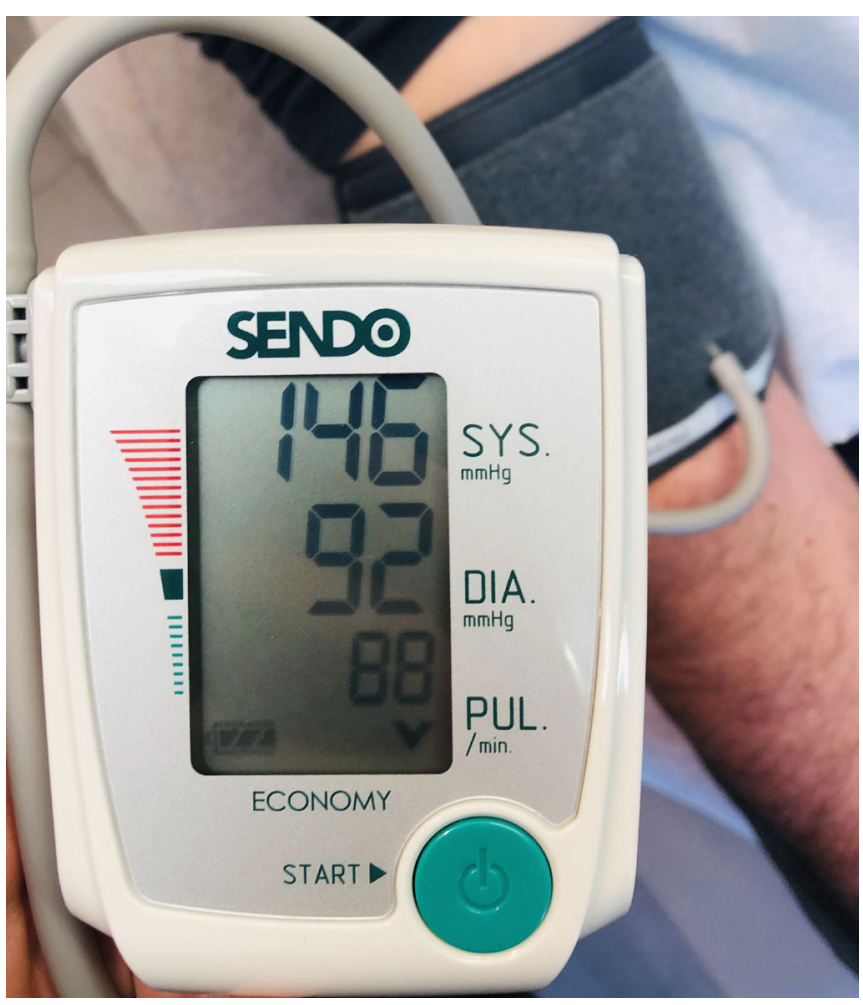

\section{Body temperature}

Body temperature is a vital sign that provides some information regarding the overall metabolic status of the patient. Its use in routine dentistry is restricted to evaluating patients for fever before dental treatment.

Core temperature: The tympanic membrane provides an accessible site for the measurement of core body temperature. Since it can be measured within a few seconds, the tympanic thermometer has become commonplace. $\mathrm{Pa}$ - 
tients receiving general anaesthesia are often monitored by oesophageal or rectal thermometers, but neither is acceptable for routine dentistry.

Skin temperature: The axilla is a good skin site for estimating core body temperature. It is, however, difficult to monitor axillary temperature continuously in the dental setting. [42]

However, this thermometer is now rapidly being replaced by faster and simpler electronic devices based on infrared thermometry, which moreover pose no threat to the environment- the infrared thermometer. (Fig. 4)

Fig. 4. Infrared thermometer

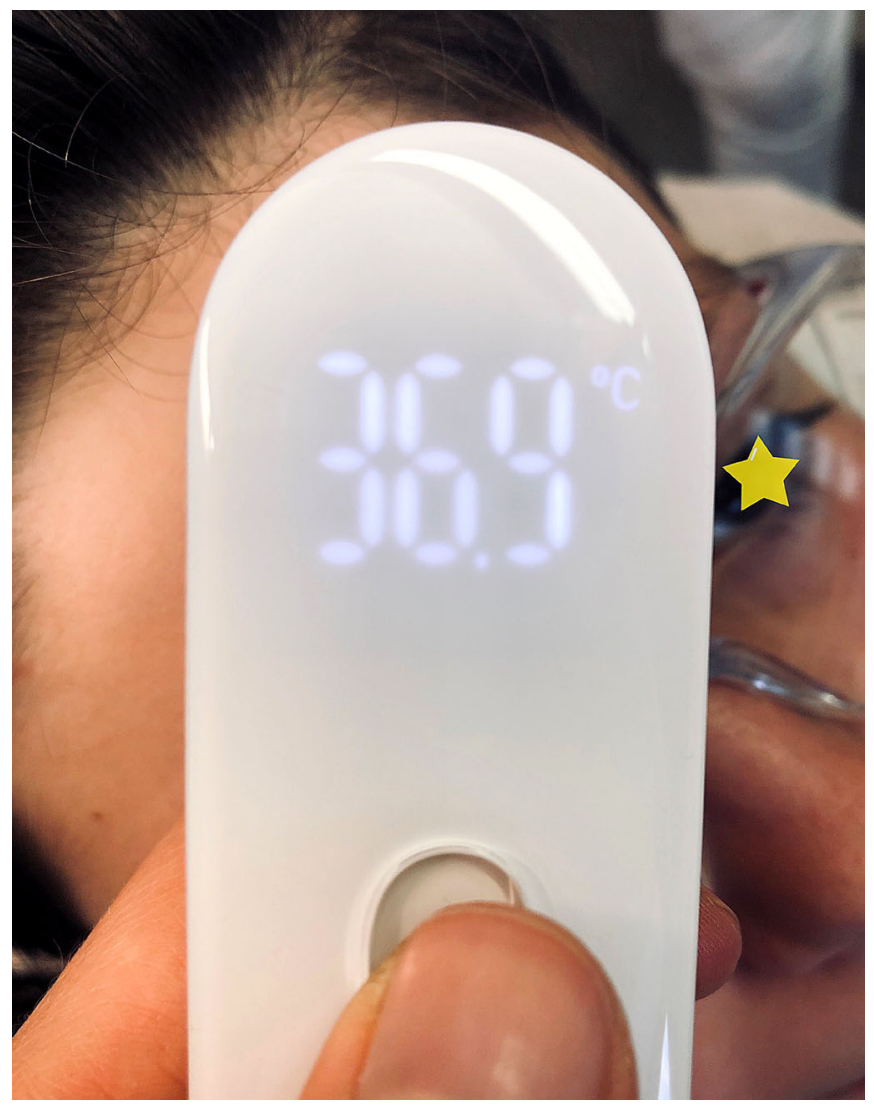

\section{DISCUSSION:}

Most of the cardiovascular changes induced during the tooth extraction and third molar surgery are within normal limits in most of the investigations, taking into consideration the comorbidity, anxiety and stress produced by the surgical intervention. $[8,9,10]$

Although the pulse oximeter is not commonly found in the dental office, it has potential value in treating medically challenged patients, especially those with advanced cardiopulmonary disorders. Application of the machine and interpretation of pulse oximetry data are so easy that not only the dentist but also the dental hygienist and assistant can use the device successfully. The pulse oximeter also serves as an excellent heart rate monitor, and devices equipped with a plethysmograph yield some information about the beat-to-beat stroke volume (which can oscillate with certain arrhythmias). Finally, the monitor itself is reliable, relatively small, and increasingly inexpensive.

The precordial stethoscope and the capnograph represent opposite extremes in cost with respect to monitoring ventilation. Although a primitive monitor by modern standards, the precordial stethoscope is the only device that provides instantaneous information regarding airway patency, ventilatory effort, and cardiac rate and rhythm. A major problem in the conscious patient is that verbal responses may be deafeningly loud. Unfortunately, encouraging patient silence potentially limits the basic assessment of neurologic function. The capnograph avoids this limitation but remains problematic for most dental offices because of its dubious cost/benefit ratio. An example where the benefits of the precordial stethoscope or capnograph may outweigh their limitations is in the patient with advanced dementia who is incapable of verbal responses on command. [4] The females showed higher levels of anxiety. The most anxious patients had the lowest BP values and the highest HR, although the differences did not reach statistical significance. The variations in BP and HR during surgical extraction of the molars were within normal limits. In the case of BP, no significant changes were recorded; the highest mean SBP and DBP values were observed at the time of ostectomy and/or tooth sectioning. The lowest HR values were recorded at baseline, before the start of the surgical procedure, whereas the highest values were obtained during incision and flap raising. [1, 9, 10]

Heart rate and blood pressure alterations during extraction can be attributed to the occurrence of trigeminocardiac reflex and should be monitored even during simple extraction procedures as the possibility of developing bradycardia, and its subsequent syncope exists. [2]

Administration of $4 \%$ articaine with $1: 100,000$ epinephrine was safe during tooth extraction procedure with no statistical difference in haemodynamic status between healthy patients and patients with controlled hypertension. Only oxygen saturation at the time of local anesthesia administration and 15 minutes after tooth extraction was higher in some patients with a statistically significant difference. [3]

\section{CONCLUSIONS:}

Monitoring of vital signs provides several benefits for clinical practice. It can help the oral surgeon to detect acute medical emergencies that may require an immediate response a well it can reveal gradual deleterious trends that can often be easily reversed before a true emergency occurs and can help in evaluating the efficacy of emergency treatments or preventive measurements that are rendered.

\section{Acknowledgement:}

National Program "Young Scientists and Postdoctoral Students", published in the State Gazette from 15. 11. 2018. 


\section{REFERENCES:}

1. Alemany- Martinez A, Valmaseda-Castellon E, Berini-Aytes L, Gay-Escoda C. Hemodynamic changes during the surgical removal of lower third molars. J Oral Maxillofac Surg. 2008 Mar;66(3):453-61. [PubMed] [Crossref]

2. Makani R, Nilesh K. Hemodynamic changes in exodontia patients using 4\% articaine with 1: 100,000 epinephrine and $2 \%$ lidocaine with 1:200,000 epinephrine. Am J Exp Clin Res. 2017; 4(3):223-228. [Internet]

3. Rashad A, Smeets R, Rana M, Bohluli B. Extraction bradycardia: a pilot case-crossover study. Head Face Med. 2013 Oct 17;9:29. [PubMed] [Crossref]

4. Fukayama H, Yagiela JA. Monitoring of vital signs during dental care. Int Dent J. 2006 Apr;56(2):1028. [PubMed] [Crossref]

5. Lima AP, Beelen P, Bakker J. Use of a peripheral perfusion index derived from the pulse oximetry signal as a noninvasive indicator of perfusion. Crit Care Med.2002 Jun;30(6):12103. [PubMed] [Crossref]

6. De Felice C, Latini G, Vacca P, Kopotic RJ. The pulse oximeter perfusion index as a predictor for high illness severity in neonates. Eur $J$ Pediatr.2002 Oct;161(10):561-2. [PubMed] [Crossref]

7. Yagiela JA, Turner RN. Hypertension. In: Medical Emergencies in Dentistry. 1st Edition. Bennett JD, Rosenberg MB, Eds. Saunders. 15th October 2001.

8. The sixth report of the Joint Na- tional Committee on prevention, detection, evaluation, and treatment of high blood pressure. Arch Intern Med. 1997 Nov 24;157(21):2413-46. [PubMed] [Crossref]

9. Tarazona-Alvarez P, PellicerChover H, Tarazona-Alvarez B, Penarrocha-Oltra D, Penarrocha-Diago M. Hemodynamic variations and anxiety during the surgical extraction of impacted lower third molars. J Clin Exp Dent. 2019 Jan 1;11(1):e27-e32. [PubMed] [Crossref]

10. Gadve VR, Shenoi R, Vats V, Shrivastava A. Evaluation of Anxiety, Pain, and Hemodynamic Changes during Surgical Removal of Lower Third Molar under Local Anesthesia. Ann Maxillofac Surg. 2018 Jul-Dec; 8(2) :247-253. [PubMed] [Crossref]

Please cite this article as: Tomeva N, Deliverska E. Monitoring of vital signs and hemodynamic changes in patients undergoing tooth extraction and third molar surgery - literature review. J of IMAB. 2020 Apr-Jun;26(2):3087-3091. DOI: https://doi.org/10.5272/jimab.2020262.3087

Address for correspondence:

Natali Tomeva

Department of Oral and Maxillofacial surgery, Faculty of Dental Medicine, Medical University Sofia,

1, Georgi Sofiiski Blvd., 1431 Sofia, Bulgaria.

E-mail: ntomeva@abv.bg 